\title{
Sub-Nyquist sampling for short pulses with Gabor frames
}

\author{
Cheng Wang ${ }^{1 *}$, Ying Wang ${ }^{2}$, Peng Chen ${ }^{3}$, Chen Meng ${ }^{1}$, Xiangjun Song ${ }^{3}$ and Wanling $\mathrm{Li}^{3}$
}

\begin{abstract}
For analog signals comprised of several, possibly overlapping, finite duration pulses with unknown shapes and time positions, an efficient sub-Nyquist sampling systems is based on Gabor frames. To improve the realizability of this sampling system, we present alternative method for the case that Gabor windows are high order exponential reproducing windows. Then, the time translation element could be realized with exponential filters. In this paper, we also construct the measurement matrix and prove that it has better coherence than Fourier matrix. Besides, for satisfying restricted isometry property (RIP), we reduce the row number and the sparsity by stretching windows and raising E-spline smoothness order. We deduce the signal reconstruction error bound, proving that appropriate selection of the stretching factor and smoothness order guarantees low reconstruction error. At last, we also provide the error bound in presence of noise, showing that the sampling scheme holds nice robustness with high Gabor frames redundancy.
\end{abstract}

Keywords: Compressed sensing, Gabor frames, Exponential reproducing windows, Sub-Nyquist sampling, Xampling

\section{Introduction}

Sub-Nyquist sampling, which can acquire signals even at very low sampling rate and yet maintain high approximation precision, has been developed over the past years to process certain signal models [1]. Unlike the traditional sub-Nyquist methods such as demodulation, under sampling ADC, and periodic nonuniform sampling, Xampling originates from CS theory (compressed sensing) and ends up in some less sophisticated front-end hardware [2-5]. Until now, a variety of different applications of Xampling is developed for multipulse signals, such as radar signals [6, 7], ultrasound signals [8], and certain signals prevalent in smart power system $[9,10]$ and smart city $[11,12]$. However, all the applications are based on finite-rate-ofinnovation (FRI) signals sampling [13, 14], and the pulse shape should be a priori known. Gabor frame sub-Nyquist sampling is proposed for making up for the weakness of FRI signals sampling and can acquire both location and shape information for multipulse signals [15]. Unlike the FRI signals sampling, which just collects the signal Fourier coefficients and reconstruct original signal with kernel functions, it operates short-time Fourier transform on signal and collects the Gabor time-frequency coefficients.

\footnotetext{
* Correspondence: 32626364@qq.com

'Shijiazhuang Mechanical Engineering College, Shijiazhuang 050003, China Full list of author information is available at the end of the article
}

Structurally, Gabor frame sub-Nyquist sampling scheme gets a little more closer to the time domain edition of modulated wideband converter (MWC) [16], which cuts the frequency domain into many lattices and measures the linear compressive translations [17]. It cuts the time domain with modulated window sequences, namely Gabor frames, and recovers exact time locations and shape with CS algorithms.

We focus here on a class of multipulse signals, which have limited time duration and short pulses with arbitrary shapes and positions, and may even overlap. The only parameters assumed are the duration $T$, the number $N_{p}$ of pulses, and the maximal width $W$. The multipulse signal, $x(t)$, supported on the interval $[-T / 2, T / 2]$, which can be expressed as

$$
x(t)=\sum_{n=1}^{N} h_{n}(t), \text { where } \max _{n}\left|\operatorname{supp}_{n}\right| \leq W
$$

If $\epsilon_{\Omega}<1$, a signal with $\epsilon_{\Omega}$-bandlimited to $F=[-\Omega / 2, \Omega / 2]$, named as essential band, is defined as

$$
\left(\int_{F^{c}}|\hat{x}(i f)|^{2} d f\right)^{1 / 2} \leq \epsilon_{\Omega}\|x(t)\|_{2}
$$

where $\hat{x}($ if $)$ denotes the Fourier transform of $x(t)$ and the symbol $F^{c}$ the complement of the set $F$. 
Theoretically, all square-integrable time-limited signals can be well approximated by truncated Gabor series. Under Gabor sampling scheme, the sampling rate equals $1 / T$ and the sampling number is about only $4 \mu^{-1} \Omega^{\prime} W N_{p}$, where $\Omega^{\prime}$ is related to the essential bandwidth of the signal and $\mu \in(0$, 1 ) is the redundancy of the Gabor frames used for processing. The sampling scheme in [15] is demonstrated to possess great reconstruction performance with time domain modulation measurement functions constructed by Bernoulli random matrix and Gabor window sequences, such as piecewise spline or B-spline window sequences. Unfortunately, there still exists a gap between the theory and practice, because the shifting Gabor windows modulated by random measurement matrix is hard to be realized with simple circuit, and its complexity and synchronization precision also greatly affect the reconstruction performance.

The first and main contribution of this paper is introducing the exponential reproducing windows into Gabor sampling scheme and reducing the time domain modulation functions, constructed by appropriately weighted window sequences, to simple exponential functions. The time domain modulation functions of this study can avoid holding complicated function structures and intricate system functions, which are difficult to realize in real world. Any time domain response function from E-spline system could be defined as exponential reproducing function, the simplest one being E-spline itself [18]. Interestingly, with appropriate weighting coefficients, the exponential reproducing window sequences can be synthesized to a simple exponential function. It could be viewed as the impulse response representation of an exponential filter, which can be carried out with simple passive electric circuit $[19,20]$. For this study, we deduced the representation of the filter form of the time domain modulation functions in Gabor sampling scheme and chose the appropriate E-spline parameter vector $\alpha$ for guaranteeing the window positive real functions. Then, we constructed the measurement matrix for CS recovery and calculated each entry and proved that such measurement matrix has better coherence than that of DFT matrix. Hence, the measurement matrix satisfies the RIP, making it possible to recover the sparse Gabor coefficient matrix perfectly.

Next, we study the effect of frame window width $W_{g}$ on the signal reconstruction performance. In [15], the frame window width is the same as the pulse width of the signal to be measured and the Gabor frames is not quite redundant. So the sparsity $S$ of the Gabor coefficients recovered for signal representation is very small and the column dimension to row number ratio $r$ of the measurement matrix is large enough to result in good RIP. However, to ensure enough sampling channel number in the sampling scheme proposed here, the E-spline smoothness order $N$ should not be too small. According to [21], if $N$ is large, which means the frame windows are high order exponential reproducing windows, the frames will be very redundant, and so the RIP may be hard to be satisfied [22]. In this study, we find that, for neutralizing the disadvantage caused by the redundancy, the frame windows width $W_{g}$ could be stretched wider to bring down $S$ and increase $r$, enhancing the measurement matrix RIP. Then, we deduce the signal reconstruction error bound, proving that it is effective for improving signal reconstruction performance.

As a third contribution, we study the robustness of the proposed sampling scheme. We deduce the signal reconstruction error bound with noise injected to the sampling scheme. From the error bound representation, we discover that when $N$ is raised to a bigger number, the redundancy would be $\mu \ll 1$, which enables the Gabor frames to hold good robustness. If the sampling channel numbers are the same, our sampling scheme can suppress noise better than in [15].

\section{Background and problem formulation}

\subsection{Gabor sampling scheme}

For any functions $x(t), g(t) \in L_{2}(\mathbb{R})$, whose modulation and translation operators are defined as $M_{b l} x(t)=$ $e^{2 \pi i b l t}$ and $T_{a k} x(t)=x(t-a k)$, there exist constants 0 $<A \leq B<\infty$, making a collection $\mathcal{G}(g, a, b)=$ $\left\{M_{b l} T_{a k} g(t)=e^{2 \pi i b l t} g(t-a k) ; k, l \in \mathbb{Z}\right\}$ satisfy

$$
A\|x\|^{2} \leq \sum_{k, l \in Z}\left|\left\langle x, M_{b l} T_{a k} g\right\rangle\right|^{2} \leq B\|x\|^{2},
$$

then $\mathcal{G}(g, a, b)$ is a Gabor frame. If we define Gabor frame coefficients as $z_{k l}=V_{g} x(a k, b l)=\left\langle x, M_{b l} T_{a k} g\right\rangle$, then signal $x(t)$ can be expanded with Gabor frames as

$$
x=\sum_{k, l \in Z} z_{k, l} M_{b l} T_{a k} \gamma
$$

In Eq. (4), $\gamma(t)$ is the dual window of $g(t)$ and $\mathcal{G}(\gamma, a, b)$ is the corresponding dual frame. Generally, if $g(t)$ is compactly supported on some interval $\left[-W_{g} / 2, W_{g}\right]$, with $a=\mu W_{g}, b=1 / W_{g}$ for some $\mu \in(0,1)$, the frame operator takes on the particularly simple form $S(t)=\sum_{k=z}|g(t-a k)|^{2}$, and the canonical dual will be $\gamma(t)=b S^{-1} g(t)$ [23]. In addition, here there exists $\gamma(t) \in S_{0}$, where $S_{0}$ is the Segal algebra space, defined as [24]

$$
S_{0}:=\left\{x \in L_{2}(\mathbb{R}) \mid\|x\|_{S_{0}}=\left\|V_{\varphi} x\right\|_{L^{1}(\mathbb{R} \times \hat{\mathbb{R}})}<\infty\right\}
$$

In the sampling scheme, the signal $x(t)$ enters $J \times M$ channels simultaneously. In the $(j, m)$ th channel, $x(t)$ was multiplied by a function $q_{j, m}(t)$ and processed by an integrator. The structure of the scheme is shown in Fig. 1. 


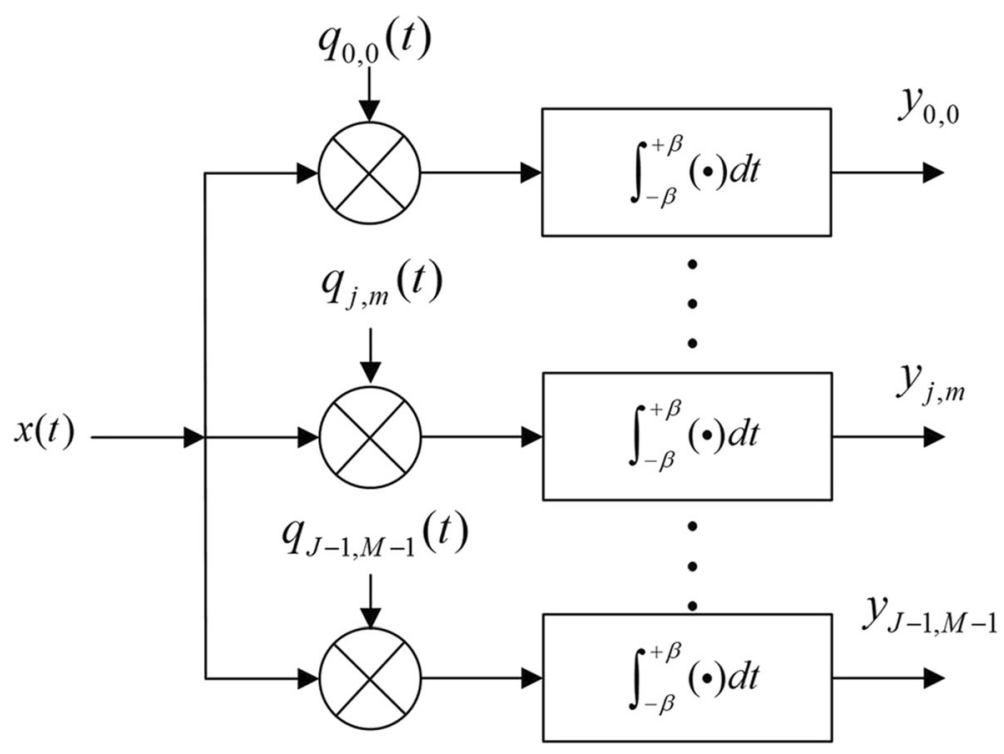

Fig. 1 Gabor sampling scheme for multipulse signals

Matusiak and Eldar [15] set $W_{g}=W$, and $g(t)$ essentially bandlimited to $\left[-B_{g} / 2, B_{g} / 2\right]$. For some, $\mu \in(0,1)$, $q_{j, m}(t)=w_{j}(t) s_{m}(t)$ is defined, where $w_{j}(t)=\sum_{l=-L_{0}}^{L_{0}} d_{j l} e^{-2 \pi i b l t}$ $s_{m}(t)=\sum_{k=-K_{0}}^{K_{0}} c_{m k} \overline{g(t-a k)}$. Here, $K_{0}=\left\lceil\frac{T+W}{2 W \mu}\right\rceil-1$ and $L_{0}$ $=\left\lceil\frac{(\Omega+B) W}{2}\right\rceil-1$, and then the numbers of time and frequency shifts are respectively $K=2 K_{0}+1$ and $L=2 L_{0}+$ 1 . The output of the $(j, m)$ th channel is

$$
y_{j, m}=\int_{-T / 2}^{T / 2} x(t) q_{j, m}(t) d t=\sum_{l=-L_{0}}^{L_{0}} d_{j l} \sum_{k=-K_{0}}^{K_{0}} c_{m k} z_{k l}
$$

Equation (6) can be written in the matrix equation as

$$
\mathbf{Y}=\mathbf{D U}^{\mathrm{T}} \text {, with } \mathbf{U}=\mathbf{C Z}
$$

For general multipulse signals, matrix $\mathbf{D}$ was used only to simplify hardware implementation, but not to reduce sampling rate. Matusiak and Eldar [15] chose matrix $\mathbf{D}$ as $\mathbf{D}=\mathbf{I}$ or other full bank matrix with $J \geq L$, reducing the primary mission of Eq. (7) to $\mathbf{U}=\mathbf{C Z}$. Relying on ideas of CS, the signal can be recovered from a small number of samples by exploiting its sparsity. Then, the multipulse signal $x(t)$ can be recovered according to Eq. (4).

Recovering matrix $\mathbf{Z}$ is referred to as a multiple measurement vector (MMV) problem [21]. In [15], matrix $\mathbf{C}$ was chosen as Gaussian or Bernoulli random matrices, which have RIP of the order $S$, if $M \geq 2\left\lceil 2 \mu^{-1}\right\urcorner N \log (K /$ $\left.2\left\lceil 2 \mu^{-1}\right\urcorner N\right)$. The RIP is defined as follows.

For every $S$-sparse vector $x$, matrix $\Phi$ has the $\left(S, \delta_{S}\right)$ restricted isometry property (RIP), if

$$
\left(1-\delta_{S}\right)\|x\|^{2} \leq\|\Phi x\|^{2} \leq\left(1+\delta_{S}\right)\|x\|^{2}
$$

for the smallest constant $\delta_{S}$, which is called restricted isometry constant (RIC).

\subsection{Exponential reproducing windows}

An exponential reproducing window is any function $g(t)$ that, together with its shifted versions, can generate complex exponentials of the form $e^{\alpha_{n} t}$, such as

$$
\sum_{k \in Z} v_{n, k} g(t-k)=e^{\alpha_{n} t}
$$

where $n=0,1, \ldots, N$, and $\alpha^{n} \in \mathbb{C}$. The coefficients are given by representation $v_{n, k}=\int_{-\infty}^{\infty} e^{\alpha_{n} t} \gamma(t-k) d t$. Knowing that the coefficients $v_{n, k}$ are discrete-time exponentials, we express them in another form

$$
v_{n, k}=\int_{-\infty}^{\infty} e^{\alpha_{n} t} e^{\alpha_{n} k} \gamma(t) d t=e^{\alpha_{n} k} v_{n, 0}
$$

The theory relating to the reproduction of exponentials derives from the concept of E-splines [18]. A function with the time domain representation $\beta_{\alpha}=e^{\alpha}$ $\operatorname{rect}\left(t-\frac{1}{2}\right)$ is called cardinal E-spline of first order. Through convolution of $\beta_{\alpha}$, Nth order E-splines can be obtained, e.g., $\beta_{\boldsymbol{\alpha}}(t)=\left(\beta_{\alpha_{1}} * \beta_{\alpha_{2}} * \cdots * \beta_{\alpha_{N}}\right)(t)$, where $\boldsymbol{\alpha}$ $=\left(\alpha_{1}, \alpha_{2}, \ldots, \alpha_{N}\right)$, and it can be written in the Fourier domain as $\hat{\beta}_{\mathbf{\alpha}}(\omega)=\prod_{n=1}^{N} \frac{1-e^{\alpha_{n}-j \omega}}{j \omega-a_{n}}$. As exponent $\alpha_{n}$ can be complex, implying that E-splines may not be real, we can avoid this case by choosing complex conjugate 
exponents. The function $\beta_{\alpha}$ is of compact support, and a linear combination of its shifted versions $\beta_{\alpha}(t-k)$ reproduces the exponential $e^{\alpha}$. Besides E-splines, any function $g(t)=\psi(t) * \beta_{\alpha}(t)$ holds the property of reproducing exponentials in the subspace spanned by $\left\{e^{\alpha_{1}}, e^{\alpha_{2}}, \ldots, e^{\alpha_{N}}\right\}$.

\section{Sampling scheme with exponential reproducing windows}

We now present a sampling scheme with exponential reproducing windows that can greatly simplify the time domain transform function $s_{m}(t)$ using the exponential reproducing property. Primarily, compared with the time duration $[-T / 2, T / 2]$ set in [15], shifting the time duration to a complete positive scope $[0, T]$, E-splines are generally defined in a positive domain. For short pulse stream, it satisfies that $W N_{p} \ll T$ and matrix $Z$ is sparse.

\subsection{Exponential reproducing transform windows}

According to Section 2.2, exponential reproducing windows $g(t)=\psi(t)^{*} \beta_{\alpha}(t)$ is considered as response to sampling kernel function $\psi(t)$ filtered with E-splines $\beta_{\alpha}(t)$. Under ideal conditions, here let $\psi(t)=\delta(t)$, the windows is reduced to $g(t)=\beta_{\alpha}(t)$. Operating a time scaling on $N$ th order cardinal E-spline function $\beta_{\boldsymbol{\alpha}}(t)$ with factor $N / W_{g}$, the Gabor window and its dual window can be expressed as $g(t)=\beta_{\mathbf{\alpha}}\left(t N / W_{g}\right)$

Then, Gabor window $g(t)$ is compactly supported in interval $\left[0, W_{g}\right]$. In this scenario, the lattice parameters are $a=\mu W_{g}$ and $b=1 / W_{g}$. If we let $\mu=1 / N$, the following equation is obtained

$$
\sum_{k \in Z} v_{n, k} g(t-k)=\sum_{k \in Z} v_{n, k} \beta_{\mathbf{\alpha}}\left(t N / W_{g}-k\right)=e^{\alpha_{n} N t / W_{g}}
$$

In fact, the time duration of the signal is $[0, T]$ and the sampling rate is restricted to $1 / T$, so that the window sequence can be truncated. To ensure that the exponential functions constructed by the shift windows can cover $[0, T]$ in time domain, they were calculated by assuming the lower and upper shift counts limit as $K_{1}$ and $K_{2}$ :

$$
\begin{gathered}
K_{1} a \geq-W_{g} \\
K_{2} a \leq T+W_{g}
\end{gathered} \rightarrow K_{2}=\left[\frac{\left(T+W_{g}\right) N}{W_{g}}\right\rceil_{-1}
$$

Equation (12) shows that the time domain is divided into $K=K_{2}-K_{1}$. Then according to Eq. (11), the time domain transform function is

$$
s_{m}(t)= \begin{cases}e^{\overline{\alpha_{n}} N t / W_{g}} & t \in[0, T] \\ 0 & t \notin[0, T]\end{cases}
$$

\subsection{Filter form}

Equation (13) has an exceedingly simple form, as compared with that of the general settings discussed in [15]. Nonetheless, $\mathbf{s}_{m}(\mathrm{t})$ is implemented with a filter.
First of all, construct function $X_{m}^{*}(t)=\sum_{p=K_{1}}^{K_{2}} v_{m, p}$ $\overline{\beta_{\boldsymbol{\alpha}}\left(-t N / W_{g}+\mu N(p-1)\right)}$ here. Given that $\mu=1 / N, p$, $q \in\left\{K_{1}, K_{1}+1, \ldots, K_{2}\right\}, p+q=K-2 N$, and the integral interval is restricted to $[0, T]$, let $X_{m}(t)=X_{m}^{*}(t)$ rect $(t-T / 2)$. Then Eq. (6) can be transformed as

$$
\begin{aligned}
y_{j, m} & =\int_{0}^{T} x(t) w_{j}(t) s_{m}(t) d t \\
& =\int_{0}^{T} x(t) w_{j}(t) \sum_{q=K_{1}}^{K_{2}} c_{m, q} \overline{\beta_{\boldsymbol{\alpha}}\left(t N / W_{g}-q\right)} d t \\
& =\int_{0}^{T} x(t) w_{j}(t) \sum_{p=K_{1}}^{K_{2}} c_{m, p} \overline{\beta_{\boldsymbol{\alpha}}\left(t N / W_{g}-\mu N(K-2 N-p)\right)} d t \\
& =\int_{0}^{T} x(t) w_{j}(t) \sum_{p=K_{1}}^{K_{2}} c_{m, p} \overline{\beta_{\boldsymbol{\alpha}}\left(-(\tau-t) \frac{N}{W_{g}}+\mu N(p-1)\right)} d t \\
& =\left(x(t) w_{j}(t) * X_{m}(t)\right)[\tau]
\end{aligned}
$$

Evidently, $\chi_{m}(t)$ represents the unit impulse response of the filter in the $(j, m)$ th channel. What is notable is that $y_{j m}$ is the integral result in time $\tau=W_{g}(K-2 N+1) / N$. According to Eq. (13), $\tau=W_{g}\left(K_{2}-K_{1}-2 N+1\right) / N=[T] \geq T$. Consequently, if the sampling action occurs in $t_{s}$, it suggests that the sample $y_{j, m}$ is acquired from the $(j, m)$ th channel. In addition, index $m$ corresponds to $n$ and the total number of time domain transform functions equals the order of E-splines, namely $M=N$. Figure 2 shows the transformation of Gabor sampling scheme with $s_{m}(t)$, carried out by filters other than waveforms.

By inserting Eq. (6) into the expression, $X_{m}(t)$ can be reduced to a simple form as $\chi_{m}(t)=e^{-\overline{\alpha_{m}} N t / W_{g}}$ rect $(t-T / 2)$, which is an exponential function truncated by a rectangle window supported in $[0, T]$. Then, the Laplace transform of $\chi_{m}(t)$ is expressed as

$$
X_{m}(s)=\frac{1-e^{-T\left(s+\overline{\alpha_{m}} N / W_{g}\right)}}{s+\overline{\alpha_{m}} N / W_{g}}
$$

Besides the order of the E-splines and window width, the parameter vector $\alpha$ is the adjustable variable that decides the filter characteristics. Each entry $\alpha_{m}$ consists of real and imaginary parts. For the exponent item of Eq. (15), the real part was used to simplify the expression. Choose $\alpha_{m}=\alpha_{0}+i m \lambda$, where $m=1,2, \ldots M$. To fulfill the convergence requirement of Laplace transform, it is necessary that $\operatorname{Re}[s]>-\alpha_{0}$. The optimal setting being $\alpha_{0}=$ $W_{g} / W N$, if $s=i \omega$, for $T \gg W$ and $W_{g} \gg W$, it follows that $e^{-T\left(s+\overline{\alpha_{m}} N / W\right)}=e^{-T W_{g} / W^{2}} e^{i T(m \lambda N / W-\omega)} \rightarrow 0$. Particularly, if $W_{g}=W, \alpha_{0}$ will have simpler form as $\alpha_{0}=1 / N$. In this case, the utility of $s_{m}(t)$ becomes a first order RC filter 


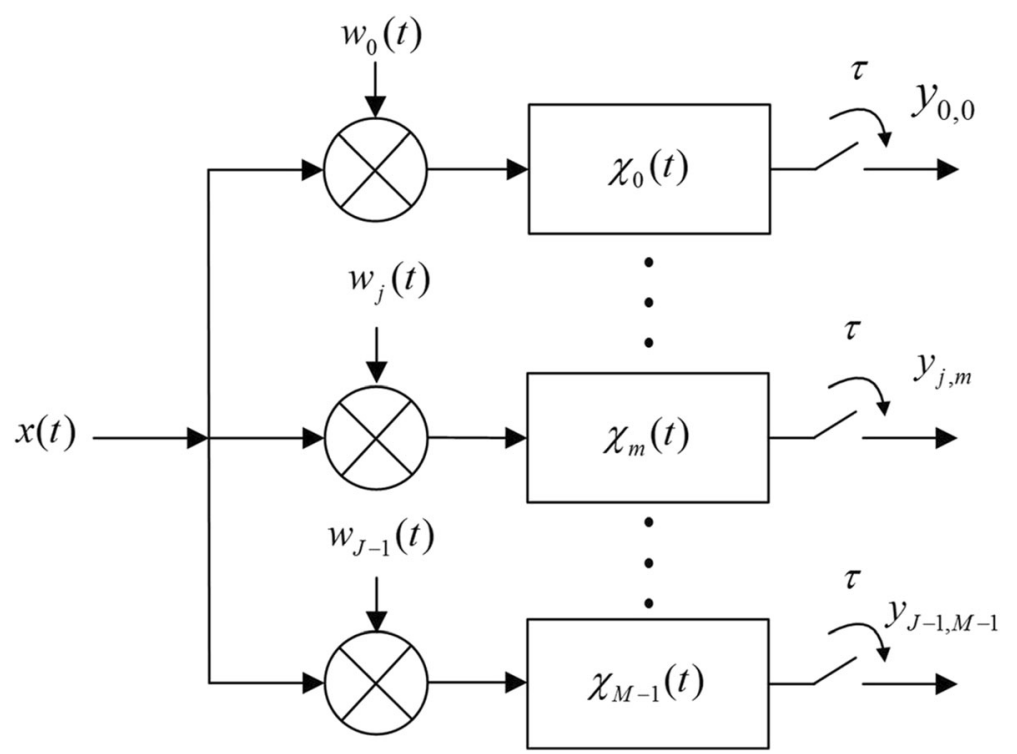

Fig. 2 Filter form of Gabor sampling scheme

$X_{m}(s)=\frac{1}{s+\overline{\alpha_{m}} N / W}$. At the same time, for $\tau=[T] \geq T$, the sample acquired in $t=t_{s}$, after passing the filter, does not lose any signal information in interval $[0, T]$.

\subsection{Signal recovery and measurement matrix}

As explained in Section 2.1, the relation between the samples $y_{j, m}$ and Gabor coefficients can be represented with matrix Eq. (7). In this paper, Eq. (7) is reduced to $\mathbf{U}=\mathbf{C Z}$, assuming in case that $\mathbf{D}=\mathbf{I}$. For every $l$, the column vectors $Z[l]$ of matrix $Z$ have only $S$ nonzero entries out of $K$, which correspond to the pulse locations. Besides, all nonzero entries are in the same rows of column vectors $Z[l]$. Matrix $Z$ can be recovered by solving MMV problem. In [22], it is suggested that, given $\mathbf{U} \in \mathbb{R}^{M \times L}$ and $\mathbf{C} \in \mathbb{R}^{M \times K}$ with $M<K$, one can find:

$$
\mathbf{Z}^{\prime}=\arg \min |\operatorname{supp} \mathbf{Z}| \text { s.t. } \mathbf{U}=\mathbf{C Z}
$$

For this study, we focus mainly on the construction of measurement matrix $C$. To bring out the concrete measurement matrix $\mathrm{c}_{m, k}$, the first and foremost task is to fix $\alpha_{m}$. For the above analysis, we have chosen $\alpha_{m}=\alpha_{0}+$ $i \lambda \xi(m)$, with $\alpha_{0}=W_{g} / N W$, and here we just need to decide $\lambda$. According to Eq. (10), $c_{m, k}=e^{\alpha_{m} k} c_{m, 0}$. If there is a component such as $2 \pi i m$ in $\lambda$, it is possible to construct a measurement matrix that has the same properties as those of Fourier matrix. So, if we fix $\lambda$ as $\lambda=2 \pi /$ $K$, then $\alpha_{m}=\frac{W_{g}}{N W}+i \frac{2 \pi \xi(m)}{K}$.

According to Section 3.2, and given that $p, q \in\left\{K_{1}\right.$, $\left.K_{1}+1, \ldots, K_{2}\right\}, p+q=K-2 N$, we can compute $\mathrm{c}_{m, k}$ as follows:

$$
\begin{aligned}
& c_{m, k}=v_{m, q}=v_{m,(K-2 N-p)} \\
& =\int_{-\infty}^{+\infty} e^{-\overline{\alpha_{m}} N t / W_{g}} \overline{\tilde{\beta}_{\boldsymbol{\alpha}}}\left(-t N / W_{g}+(K-2 N-p-1)\right) d t
\end{aligned}
$$

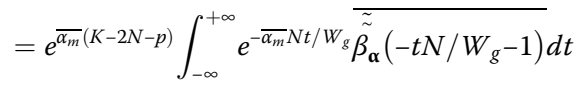

$$
\begin{aligned}
& =e^{\overline{\alpha_{m}} q} \int_{-\infty}^{+\infty} e^{-\overline{\alpha_{m} N t} / W_{g}} \overline{{\overline{\beta_{-\alpha}}}^{\sim}\left(t N / W_{g}\right)} d t \\
& \stackrel{*}{=} e^{\overline{\alpha_{m}} k} \frac{\Xi(m)}{|\Xi(m)|^{2}},
\end{aligned}
$$

where $\Xi(m)=\frac{W_{g}}{N} \prod_{n=1}^{N} \frac{1-e^{\overline{\alpha_{n}}+\overline{\alpha_{m}}}}{\overline{\alpha_{n}}+\overline{\alpha_{m}}}, k \in\left\{K_{1}, K_{1}+1, \ldots, K_{2}\right\}$.

To avoid $g(t)=\beta_{\boldsymbol{\alpha}}\left(t N / W_{g}\right)$ from being a complexvalued function, we need $\alpha$ to be a vector with $\operatorname{Im}\left(\alpha_{m}\right)+$ $\operatorname{Im}\left(\alpha_{M-m}\right)=0$, because $\beta_{\boldsymbol{\alpha}}\left(t N / W_{g}\right)$ is the convolution of functions $\beta_{\alpha_{m}}\left(t N / W_{g}\right)$ and the convolution of $\beta_{\alpha_{m}}$ $\left(t N / W_{g}\right)$ and $\beta_{\alpha_{M-m}}\left(t N / W_{g}\right)$ generates a real value. So for $\operatorname{Im}\left(\alpha_{m}\right)=i \frac{2 \pi \xi(m)}{K}, m=0,1, \ldots, M-1$, we can let

$$
\operatorname{Im}\left(\alpha_{m}\right)= \begin{cases}i \frac{2 \pi}{K}(2 m-M+1) & M \text { is even } \\ i \frac{2 \pi}{K} \frac{2 m-M+1}{2} & M \text { is odd }\end{cases}
$$

Until now, we have deduced the representation of $\mathrm{c}_{m, k}$, which is the $(m, k)$ th entry of measurement $C$. And, we also determine the parameter vector $\alpha$, with the $m$ th entry expressed as $\alpha_{m}=\frac{W_{g}}{W N}+i \frac{2 \pi \xi(m)}{K}$.

However, for measurement of CS recovery, $C$ should satisfy the RIP. We can see that $C$ is a weighted DFT matrix. 
As there is still no effective and easy method to compute the RIC of such kind of matrices except ergodic calculating, we detect the RIP by comparing it with DFT matrix, which was proved to satisfy RIP for sparse signal reconstruction [23]. We need to explore the matrix coherence $\theta$. And for any matrix $\Phi$, it is defined as [22]

$$
\theta(\Phi):=\max _{i \neq j} \frac{\left|\left\langle\phi_{i}, \phi_{j}\right\rangle\right|}{\overline{\left\|\phi_{i}\right\|_{2}\left\|\phi_{j}\right\|_{2}}}
$$

Then by applying Gershgorin's circle theorem, we can conclude that $\delta_{S} \leq(S-1) \theta(\Phi)$. Furthermore, a $(2 S, \delta)$ RIP matrix with $\delta_{2 S}<1$ necessarily has all subcollections of $2 S$ columns that are linearly independent, so, as [24], a normalized DFT matrix satisfies $\delta_{2 S}=(2 S-1) \theta(\Phi)$. Here, we use matrix coherence to judge the $2 S$-order RIP of matrix C. Taking Eq. (17) into Eq. (19), we get

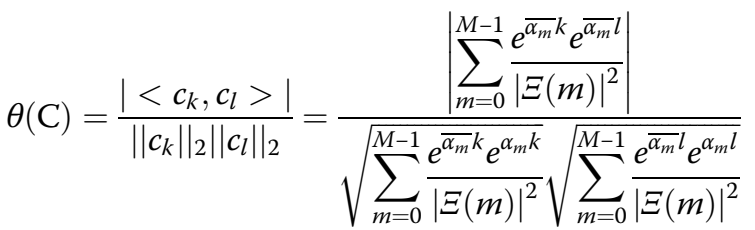

$$
\begin{aligned}
& =\frac{\left|\sum_{m=0}^{M-1} \frac{e^{i \frac{2 \pi m}{K}(l-k)}}{|\Xi(m)|^{2}}\right| \sum_{m=0}^{M-1} \mid e^{i \frac{2 \pi m}{K}(l-k) \mid}}{\sum_{m=0}^{M-1} \frac{1}{|\Xi(m)|^{2}}}=\theta\left(\mathbf{D F T}_{Q}\right),
\end{aligned}
$$

where $\theta\left(\mathbf{D F T} \mathbf{T}_{Q}\right)$ is a class of submatrices of Fourier matrices DFT, which are $K \times K$ matrices with each entry as $(\mathbf{D F T})_{m, k}=\frac{1}{\sqrt{K}} \exp \left(i \frac{2 \pi m k}{K}\right)$, and Q is an index set $\{1$, $2, \ldots, M\}$ with $M \leq K$. As a result, the weighted DFT matrix $C$ also satisfies RIP and many other properties of DFT $_{Q}$. In [22], theorem 3.3 shows that for any $t>1$ and any $K, S>2$, a random subset $Q$ of average cardinality

$$
M=(C t S \log K) \log (C t S \log K) \log ^{2} S
$$

satisfies the RIP condition with probability of at least 1 $-5 e^{-c t}$ and that the $C$ with possible indices denotes absolute constants. In any fixed probability of success such as 0.99 , then Eq. (21) yields the best known bound on the number of Fourier measurements $M=O\left(S \log ^{4} K\right)$.

With Eq. (4), we are able to finish the signal reconstruction. The atoms of the dual Gabor frames corresponding to the nonzero Gabor coefficients can construct a Gabor subspace of the signal. Consequently, it can be said that a class of signals, constructed by the same kinds of pulses, belong to a union of Gabor subspaces according to [5]. The recovery of Gabor coefficient matrix $Z$ is called subspace detection. So, for perfectly reconstructing the original signal, the critical problem is how to construct the sampling scheme with appropriate relation between $S$ and $K$.

In this study, we can solve the problem from the two entry points: the windows width $W_{g}$ and the index set of measurement matrix $C$. On the one hand, in a fixed time duration, $K$ is determined by the time shifting parameter $a$. With $W_{g}$ increasing, $K$ gets smaller. On the other hand, $W_{g}$ is intimately involved with sparsity $S$. If the windows are wide enough, the sparsity $S$ can be reduced to half of that proposed in [15]. What is more, E-splines have a special property: the higher the order $N$, the more the energy is centralized to a shorter time domain support [18]. Then the essential window width gets short and the ratio $M / s=N / s$ was brought down.

\section{Frame window width for sample number}

In sampling scheme, $K$ is the dimension of vectors $\mathbf{Z}[l]$, where there exists $K=\frac{T+W_{g}}{W_{g}} N+N-1$ from Eq. (12). Here, we represent window width as $W_{g}=\zeta W, \zeta \geq 1$, then $a=\zeta W / N$ and $K=\left(\frac{T}{\zeta W}+2\right) N-1$. We can see that the larger the $\zeta$ is increased, the smaller the $K$ is. In [15], there exists $W_{g}=W$, and so $K^{\prime}=\left(\frac{T}{W}+2\right) N-1$. For $T \gg$ $W$, we can greatly reduce $K$ by this way.

In another parameter, the sparsity is represented as $S$ $=\left[\frac{W_{g}+W}{a} N_{p}\right]$. As discussed in [15], $W_{g}=W$ and $S$ $=\left[2 \mu^{-1}\right]=2 N_{p} N$. Here, forming $W_{g}=\zeta W$, we get $S=\frac{\zeta+1}{\zeta}$ $N_{p} N \leq 2 N_{p} N$. If $W_{g} \gg W, S \approx\left[\mu^{-1}\right] N_{p}=N_{p} N$. As we can see, wider window width also means smaller sparsity $S$.

For more accurate analysis, we take a good property of E-splines into account: the higher the order $N$, the more the energy is centralized to a shorter time domain support [18]. Here, introducing the concept of "essential frame windows width," we say that $\phi(t)$ has $\epsilon_{W}$-essential window width $W_{E}=\left|T_{E}\right|$, when $T_{E}=\left[t_{1}, t_{2}\right]$, where $0 \leq t_{1}<t_{2} \leq W_{g}$. Defining $T_{E 1}^{c}=\left[0, t_{1}\right], T_{E 2}^{c}=\left[t_{2}, W_{g}\right]$, if for some $\epsilon_{W}<1$

$$
\left(\int_{T_{E 1}^{c}+T_{E 2}^{c}}|\varphi(t)|^{2} d t\right)^{1 / 2} \leq \epsilon_{W}\|\varphi(t)\|_{2}
$$

With the increase in the order $N$, the $\epsilon_{W}$-essential windows width $W_{E}$ gets shorter. Defining essential window width factor $\eta=W_{E} / W_{g}$, we can measure precisely how much of the window width has been cut down, with the reserved part still holding most energy. When the order $N$ of E-spline is high enough, the essential window width factor $\eta$ becomes very small, whereas stricter $\epsilon_{W}$ means wider scope 
of permissible $\eta$. Based on the foregoing assumption, the sparsity can be described as $S=\left[\frac{W_{E}+W}{a} N_{p}\right]=\frac{\eta \zeta+1}{\zeta} N_{p} N$.

Then, the practical sparsity get smaller. As a result, to ensure that $C$ satisfy RIP, we need $M=O$ $\left(\frac{\eta \zeta+1}{\zeta} N_{p} N \log ^{4}(T N / \zeta W)\right)$. Note, in this study, $M=N$. So, for perfect subspace detection, if there exists constant $C^{\prime}>0$, the following equation must be satisfied:

$$
\frac{\eta \zeta+1}{\zeta} N_{p} N \log ^{4}(T N / \zeta W) \leq \frac{1}{C^{\prime}}
$$

For matrix $C$, the ratio $M / K \approx W / T_{T}$ is mainly decided by $\zeta$. So, larger $\zeta$ means $C$ has better orthogonality and better RIP. In addition, if we enlarge $\zeta$, Eq. (23) is easier to be satisfied. As the channel number of the sampling scheme is decided by $M$, we can increase $N$ to acquire more measured samples, which means better reconstruction performance. As $\eta$ and $N$ are inversely correlated in Eq. (23), sometimes we need not to worry that $N$ is too large to prevent Eq. (23) from being satisfied.

Next, we will analyze the effect of stretching the frame windows width on the signal reconstruction error. First, we offer a lemma here.

4.0.0.1 Lemma 1 For any Gabor window functions $g(t) \in S_{0}$, if there exists a constant $0<\zeta<\infty$, we have $\left\|g\left({ }^{t} / \zeta\right)\right\|_{S_{o}}=\zeta\|g(t)\|_{S_{0}}$, and $g\left({ }^{t} / \zeta\right) \in S_{0}$.

4.0.0.2 Proof If $g^{\prime}(t)=g(t / \zeta)$, then its Fourier transformation is as the following equation

$$
V_{g^{\prime}} g^{\prime}(\tau, f)=\zeta \int_{-\infty}^{\infty} e^{-\frac{2 \pi i \omega t}{\zeta}} g\left(\frac{t}{\zeta}\right) g\left(\frac{t}{\zeta}-\tau\right) d \frac{t}{\zeta}=\zeta V_{g} g(\tau, f)
$$

Then

$$
\left\|g^{\prime}(t)\right\|_{S_{0}}=\int_{-\infty}^{\infty} \int_{-\infty}^{\infty}\left|V_{g^{\prime}} g^{\prime}(\tau, f)\right| d \tau d f=\zeta \int_{-\infty}^{\infty} \int_{-\infty}^{\infty}\left|V_{g} g(\tau, f)\right| d \tau d f=\zeta\|g(t)\|_{S_{0}}
$$

According to the definition of Segal algebraic space, for any $g(t) \in S_{0}, \quad\|g(t)\|_{S_{0}}<\infty$ is able to be satisfied under condition $0<\zeta<\infty$. So $\left\|g^{\prime}(t)\right\|_{S_{0}}=\zeta\|g(t)\|_{S_{0}}<\infty$ and $g(t / \zeta) \in S_{0}$. Prove up.

According to the lemma, if the windows are stretched $\zeta$ times, the $S_{0}$-norm is proportional to the stretching factor. What is surely guaranteed is that the $S_{0}$-norm does not relate to the size of the grid in time and frequency plane and the redundancy. Consequently, we can propose the following theorem about the signal reconstruction error bound.

4.0.0.3 Theorem 2 Given that $x(t)$ is a finite duration signal supported on the interval $[0, \mathrm{~T}]$ with $\epsilon_{\Omega}$-bandlimited
$[-\Omega / 2, \Omega / 2]$, and $\mathcal{G}(g, a, b)$ is a frame with each atom $g(t)$ supported on $[0, \mathrm{~W}]$ and $\epsilon_{B}$-bandlimited on $[-B / 2, B / 2]$, of which the dual atom $\gamma(t) \in S_{0}$. For each atom $g^{\prime}(t)=g(t / \zeta)$, with its dual atom $\gamma^{\prime}(t) \in S_{0}$, and $\epsilon_{B}>0$, there exist $K_{1}<$ $0, \mathrm{~K}_{2}>0$ and $\mathrm{L}_{0}>0$, depending on $\gamma(t)$ and the essential bandwidths of $g(t)$, the following inequality is satisfied

$$
\left\|x-\sum_{k=K_{1}}^{K_{2}} \sum_{l=-L_{0}}^{L_{0}} z_{k, l} M_{b^{\prime} l^{\prime}} T_{a^{\prime} k} \gamma^{\prime}\right\|_{2} \leq C_{0}\|x\|_{2}+C_{1}\left\|Z-Z_{\Lambda}\right\|_{2,1}
$$

where $a^{\prime}=\zeta a, b^{\prime}=b / \zeta, C_{0}=\zeta C_{\zeta}^{2}\left(\zeta\left(\epsilon_{\Omega^{-}} \epsilon_{B} \epsilon_{W}\right)+\sqrt{\zeta}\left(\epsilon_{B}+\epsilon_{W}\right)\right)\|\gamma\|_{S_{0}}\|g\|_{S_{0}}$ and $C_{1}=\zeta C_{\zeta} C_{1}\|\gamma\| S_{0}$ with $C_{\zeta}=(1+1 / \zeta a)^{1 / 2}(1+\zeta / b)^{1 / 2}, Z_{\Lambda}$ is the best $S$ row approximation of $Z$, with $|\Lambda|=S$.

The proof is rooted [15] with appropriate adjustments according to Lemma 1.

Theorem 2 shows that the reconstruction error is comprised of two parts. The first part comes from Gabor series truncating and window cutting. For Gabor windows $g(t)$ supported on a quite short time interval, $C_{\zeta} \approx\left(1+\frac{1}{\zeta W N}\right)^{\frac{1}{2}} \gg 1$.. With $\zeta$ increasing, $C_{\zeta}$ decreases evidently and $C_{0}$ is brought down. Meanwhile, the higher the E-spline smoothness order $N$ is, the smaller $C_{\zeta}$ is, which results in the error reduction. The second part $\zeta$ comes from subspace detection error. As analyzed, larger $\zeta$ means that $C$ has better RIP, which can also decrease the error. So, we can reduce the signal reconstruction error bound by enlarge window width.

The total number of Gabor coefficients is related to a somewhat larger interval $[0, T]$, where $T^{i}=T+2 \zeta W$, with $K \approx^{T^{\prime} N} / \zeta W$ in time domain, and $[0, \Omega]$, where $\Omega^{\prime}=\Omega$ $+{ }^{B} / \zeta$, with $L \approx \zeta W \Omega^{\prime}$ in the frequency domain. Overall, the required number of samples is $K L \approx \frac{T^{\prime} N}{\zeta W} \zeta W \Omega^{\prime}=T^{\prime} \Omega^{\prime}$ $N$. To decrease $K L$ further, $b$ can be maintained constant at $b={ }^{1} / W$. Then $L \approx W \Omega^{\prime}$ and the size of matrix $Z$ becomes $K L \approx T^{\prime} \Omega^{\prime} N / \zeta$. By this method, if $\zeta$ is big enough, the calculation load can be greatly reduced. Reducing Gabor coefficient numbers may enlarge the error bound, but we still can acquire acceptable error with suitable factor $\zeta$.

\section{Noisy measurements}

All the signals, hitherto considered for sampling, were noise-free or exactly multipulse. But when the signals to be measured are noisy, according to [15], the Gabor sampling scheme is robust to bounded noise in both the signal and the samples. Here, we will study the robustness of the sampling scheme proposed in this paper.

The signals are limited to the interval $[0, T]$, which means the noise or the energy leaks between the pulses are also limited. As a result, the column vectors $Z[l]$ are no longer sparse. Nonetheless, according to earlier analysis, $Z$ can be 
well approximated to a sparse $Z$, which consists of $|\Lambda|$ rows of $Z$ with largest $l_{2}$ norm, and zeros, otherwise referred to as the best $|\Lambda|$-term approximation. If the indice sets $\Lambda$ is complete, $|\Lambda|=S$. Then, Eq. (7) can be written as

$$
\mathrm{Y}=\mathrm{DU}^{T}+\mathbf{N}^{\prime} \text {, with } \mathbf{U}=\mathbf{C Z}+\mathbf{N}
$$

With $D$ having a full column rank, the relation to Eq. (25) can be reduced to $\mathbf{U}=\mathbf{C Z}+\mathbf{N}$, where $\mathbf{N}=\mathbf{D}^{\dagger} \mathbf{N}^{\prime}$. By utilizing CS algorithms, $\mathbf{Z}^{\prime}$ can be resolved well even with noisy terms, and the following inequality, satisfying [25]:

$$
\left\|\mathbf{Z}-\mathbf{Z}^{\prime}\right\|_{2} \leq C_{1}^{\prime}\left\|\mathbf{Z}-\mathbf{Z}_{\Lambda}\right\|_{2,1}+C_{2}^{\prime}\|\mathbf{N}\|_{2}
$$

where $\mathrm{C}^{\prime}{ }_{1}$ and $\mathrm{C}^{\prime}{ }_{2}$ are constants depending on the RIP constant $\delta_{2 S}$ of $\mathrm{C}$. Then, according to Theorem 2, we can deduce the error bound as follows:

$$
\left\|x-\sum_{k \in \Lambda} \sum_{k=-L_{0}}^{L_{0}} z_{k, l} M_{b^{\prime} l} T_{a^{\prime} k} \gamma^{\prime}\right\| \leq C_{0}\|x\|_{2}+C_{1}\left\|\mathbf{Z}-\mathbf{Z}_{\Lambda}\right\|_{2,1}+C_{2}\|\mathbf{N}\|_{2}
$$

where $C_{0}$ is the same as that in Theorem 2, while $C_{1}$ $=\eta \zeta \bar{C}_{\zeta} C_{1}^{\prime}\|\gamma\|_{S_{0}}$ and $C_{2}=\eta \zeta \bar{C}_{\zeta} C_{2}^{\prime}\|\gamma\|_{S_{0}}$.

Here, the third part of the error bound comes from the noise. As analyzed above, increasing $\zeta$ and $N$ is also beneficial to bring down $C_{\zeta}$ and minimize the impact of the noise. What is more, with increase in the smoothnees order $N$ of the windows, the norms $\|g\|_{S_{0}}$ and $\|\gamma\|_{S_{0}}$ decrease rapidly, which is able to make the error bound going down as well. To the contrary, if we increase $N$, the RIP of $C$ may get worse, and $C_{2}^{\prime}$, which is the convergence factor for the noise, may be enlarged. Then, the noise suppression generated by the CS iteration will be weak. However, comparing the variation trends of the several factors following $N, C_{\zeta}$ and $\|\gamma\|_{S_{0}}$ play a leading role on improving the sampling scheme robustness. If $N$ rises, the redundancy of the Gabor frames would get higher. So, the analysis happens to coincide the conclusion in Eq. (26), which indicates that higher frames redundancy causes better robustness.

\section{Simulation and discussion}

We now present some numerical experiments to illustrate the reconstruction performance of short pulses with subNyquist sampling, using the scheme proposed above.

The sampling scheme was tested on a range of multipulse signals of duration $T=20 \mathrm{~ms}$, and the pulses making up the signals were randomly chosen as a set of three different pulses: cosine, Gaussian, and B-spline of three orders. The number of pulses was varied between $N_{p}=1,3,5$, the maximum pulse width being $W=0.5 \mathrm{~ms}$. The locations of the pulses were also chosen at random. Monte Carlo method was used for simulations averaged over 500 trials. Throughout the experiments, we chose $\mathbf{D}=\mathbf{I}$ and measured the relative error $\|x-\hat{x}\|_{2} /\|x\|_{2}$.

In the first experiment, we studied the effects of stretching Gabor frame windows width on the reconstruction error. The smoothness order of the E-spline windows was chosen as a fixed number $M=N=100$. The Gabor coefficients $Z$ was acquired by solving the MMV problem with SOMP.

Figure 3 shows the relative error between the reconstructed signals and the original signals with the increase in window stretching factor $\zeta$ and the pulse number $N_{p}$. The two curved surfaces represent respectively the error variation trends under the conditions of $b=1 / W$ and $b=1 / \zeta W$. It can be seen that when $b=1 / \zeta W$ and $\zeta \geq 7$, the signals can be reconstructed with minimum error, and the variation of $N_{p}$ had little effect on the error. With increasing $\zeta$, the dimension $K$ of $Z$ significantly decreased, which means that the measurement matrix has correspondingly fewer columns, and can hence be recovered with higher accuracy. In this case, time samples satisfied $K \leq 716$; also, Eq. (21) can be easily satisfied and even more pulses reconstructed. For reducing the size of Gabor coefficient matrix $Z$, we can also choose $b=1 / W$. When $\zeta \approx 7$, the recovery error of the signal will be approximately the same as that when $b=1 / \zeta W$.

In the second experiment, we studied the effects of Espline smoothness order $N$ on the relative reconstruction error under different stretching factor $\zeta$. We chose $N_{p}=3$ with no other signal parameters not changed. The simulation was started with $N=25$, adding $N$ at uniform interval $\Delta_{N}=5$ until $N=100$, while the windows stretching factor $\zeta$ was raised from 2 to 15 . The results are shown by Fig. 4a, b. According to the figures, when $N$ was small, the error was generally large. In the case that $\epsilon_{W}=0.1$ and $N=25$, there existed $\eta \approx 0.2$, and the minimum error was on the row $\zeta=5$. When $N$ was not large enough, the measurement matrix $C$ was too flat to recover $Z$ perfectly. With increasing $N$, the optimal stretching factor was raised to about $\zeta=7$. When $N$ was large enough, with appropriate ratio $M / K$ and essential

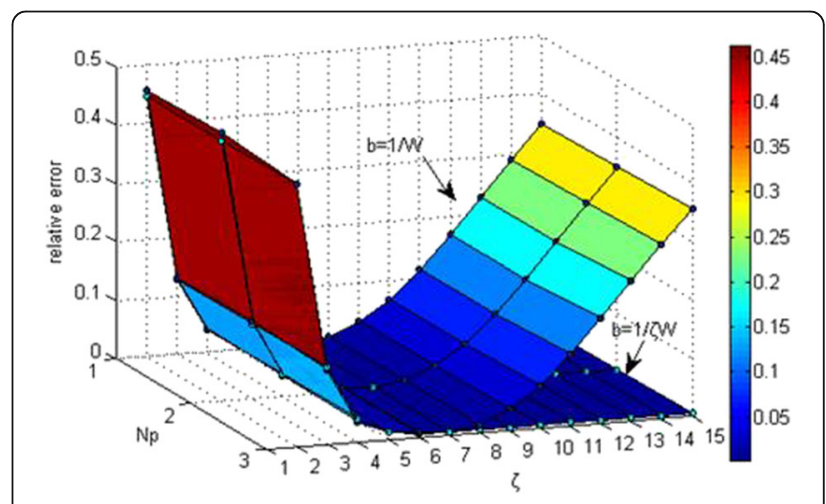

Fig. 3 Relative reconstruction error variation curve with the increasing of window stretching factor $\zeta$ and pulse number Np 


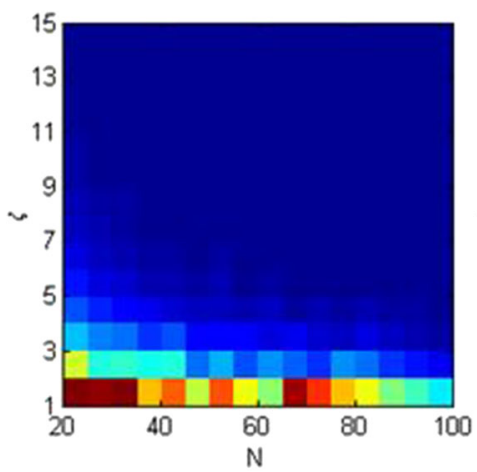

(a)

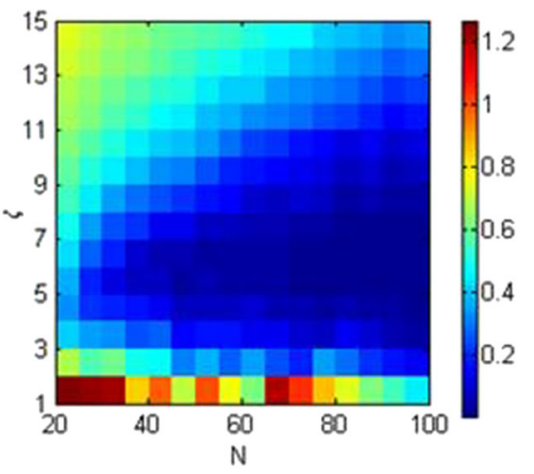

(b)

Fig. 4 Relative reconstruction error variation with increased E-spline smoothness order $N$ under different stretching factors $\zeta$. a is in the case that $b=乙 W$ when $\mathbf{b}$ is in the case that $b=1 / \mathrm{W}$

$W_{E}$, the errors became quite small, enabling broadening of the range of feasible window width.

In the third experiment, we verified the robustness of the proposed sampling scheme. In the simulation, with $N_{p}=3$, Gaussian noise with noise/signal ratio (SNR) of $15 \mathrm{~dB}$ was injected into the channel. The smoothness order $N$ was equivalent to $M$, increasing from 25 to 100 . Figure 5 shows the SNR of output signals from the sampling schemes with redundancy $\mu=1 / N$ relating to this study, and with $\mu=0.5, \mu=0.3, \mu=0.75$ relating to [15]. It was seen that for the sampling scheme proposed, the SNR increased with the raising of $N$, improved by about $20 \mathrm{~dB}$ compared with that of the input signals. When the channel numbers were the same, it was generally much higher than that of the sampling scheme in [15], showing better performance on noise suppression.

Finally, we compared the reconstruction error of the sampling scheme proposed in this paper with that of a specific example in [15]. In Table 1, Schemes I and II

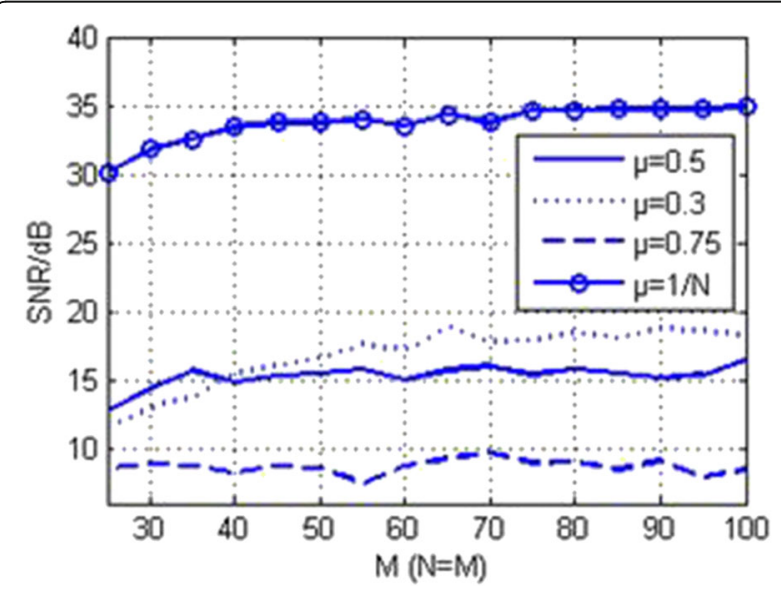

Fig. 5 SNR of the sampling schemes with redundancy as $\mu=1 / \mathrm{N}$ relating to this study, and $\mu=0.5, \mu=0.3, \mu=0.75$ relating to [15] relate respectively to the sampling schemes proposed in this paper and that in [15]. As the scheme structure changed significantly and, at the same time, the parameters $M$, $K, \mu$, and $\zeta$ could not remain the same, the comparison was restricted to some typical cases with the same signal. Here, we chose $N_{p}=3$ and set other signal parameters as they were before. Table 1 shows that, under appropriate parameter setting, the reconstruction performance of both schemes were similar. Therefore, the sampling scheme proposed here can be considered effective and feasible.

\section{Conclusions}

We introduced high order exponential reproducing windows into Gabor sampling scheme for sub-Nyquist sampling of short pulses, which notably simplified the filter's structure of the sampling system and enabled it to be realized by RC filters. Subject to choosing an appropriate E-spline parameter vector $\alpha$, the Gabor windows could be a positive real function and it is possible to construct the measurement matrix as a weighted DFT matrix for Gabor coefficients recovery, which is proved to have good coherence. Meanwhile, we developed methods for good RIP by stretching windows and increasing E-spline smoothness order $N$. With that, the ratio $r$ of the row dimension and the column dimension of the measurement matrix could be improved. Also, the sampling system was proved to hold acceptable reconstruction error bound. Because the energy of the E-splines would concentrate to a narrower support with the increasing of $N$, the Gabor coefficient matrix holds lower sparsity and hence its recovery by using CS algorithms is more reliable. For reducing the coefficients of samples further, we compressed the dual Gabor frames and proved that the reconstruction of the original signals was still under a low error bound and that it benefited from the nice robustness caused by high frames redundancy. With high frames redundancy, the sampling scheme holds nice robustness. 
Table 1 Reconstruction errors of sampling scheme proposed in this paper and that in [15]

\begin{tabular}{lllll}
\hline & Parameters & $M=50$ & $M=75$ & $M=100$ \\
\hline Scheme I & $b=1 / W, \zeta=7$ & 0.0542 & 0.0168 & 0.0023 \\
& $b=1 / W, \zeta=14$ & 0.5525 & 0.3803 & 0.2912 \\
$b=1 / \zeta W, \zeta=7$ & 0.0192 & 0.0108 & 0.0091 \\
Scheme II & $b=1 / \zeta W, \zeta=14$ & 0.0093 & 0.0091 & 0.0090 \\
& $\mu=0.3$ & 0.0053 & 0.0053 & 0.0053 \\
$\mu=0.5$ & 0.0064 & 0.0064 & 0.0064 \\
\hline
\end{tabular}

\section{Acknowledgements}

The experiments of this research were financially supported by the National Natural Science Foundation of China under Grant 61501493.

\section{Authors' contributions}

CW conceived and designed the study. PC carried out most of the analyses. CW, YW, and PC drafted the manuscript. CM, XS, and WL performed the experiments. All authors read and approved the final manuscript.

\section{Competing interests}

The authors declare that they have no competing interests.

\section{Publisher's Note}

Springer Nature remains neutral with regard to jurisdictional claims in published maps and institutional affiliations.

\section{Author details}

'Shijiazhuang Mechanical Engineering College, Shijiazhuang 050003, China.

${ }^{2}$ Tianjin University of Technology and Education, Tianjin 300222, China.

${ }^{3}$ Mechanical Technology Institute, Shijiazhuang 050003, China.

Received: 20 February 2017 Accepted: 4 April 2017

Published online: 18 April 2017

\section{References}

1. M Mishali, YC Eldar, Sub-nyquist sampling: bridging theory and practice. IEEE Signal Process. Mag. 11, 98-124 (2011)

2. J Crols, S Michiel, J Steyaert, Low-if topologies for high-performance analog front ends of fully integrated receivers. IEEE Trans. Circuits Syst. II, Analog Digit. Signal Process. 45(3), 269-282 (1998)

3. Z Li, K Liu, Y Zhao et al., MaPIT: an enhanced pending interest table for NDN with mapping bloom filter. IEEE Commun. Lett. 18(11), 1915-1918 (2014)

4. Z. Li, L. Song, and H Shi. Approaching the capacity of K-user MIMO interference channel with interference counteration scheme. Ad Hoc Networks, 2016, 46(2), Accept for Publication. DOl:10.1016/j.adhoc.2016.02.009.

5. Z Li, Y Chen, H Shi, K Liu, NDN-GSM-R: a novel high-speed railway communication system via Named Data Networking. EURASIP J Wirel. Commun. Net Work. 48, 1-5 (2016)

6. PL Dragotti, M Vetterli, T Blu, Sampling moments and reconstructing signals of finite rate of innovation: Shannon meets Strang-Fix. IEEE Trans. Signal Process. 55(5), 1741-1757 (2007)

7. X. Liu, Z. Li, P. Yang, and Y. Dong. Information-centric mobile ad hoc networks and content routing: a survey. Ad Hoc Networks, 2016, http://dx. doi.org/10.1016/j.adhoc.2016.04.005

8. R Tur, YC Eldar, Z Friedman, Innovation rate sampling of pulse streams with application to ultrasound imaging. IEEE Trans. Signal Process. 59(4), 1827$1842(2011)$

9. Z Wu, X Xia, B Wang, Improving building energy efficiency by multiobjective neighborhood field optimization. Energ Buildings 87, 45-56 (2015)

10. Z Wu, X Xia, Optimal switching renewable energy system for demand side management. Sol. Energy 114, 278-288 (2015)

11. Z Wu, TWS Chow, Binary neighbourhood field optimisation for unit commitment problems. IET Gener. Transm. Distrib. 7(3), 298-308 (2013)

12. Z Wu, X Xia, Optimal motion planning for overhead cranes. IET Control Theory Appl. 8(17), 1833-1842 (2014)
13. G. Itzhak, E. Baransky, N. Wagner, et al. A hardware prototype for subnyquist radar sensing.//Systems, Communication and Coding (SCC), Proceedings of 2013 9th International ITG Conference on. VDE, 2013: 1-6.

14. T Michaeli, YC Eldar, Xampling at the rate of innovation. IEEE Trans. Signal Process. 60(3), 1121-1133 (2012)

15. E Matusiak, YC Eldar, Sub-nyquist sampling of short pulses. IEEE Trans. Signal Process. 60(3), 1134-1148 (2012)

16. M Mishali, YC Eldar, From theory to practice: Sub-Nyquist sampling of sparse wideband analog signals. IEEE J. Sel. Topics Signal Process 4(2), 375-391 (2010)

17. YM Lu, MN Do, A theory for sampling signal from a union of subspaces. IEEE Trans. Signal Process. 56(6), 2334-345 (2008)

18. M Unser, T Blu, Cardinal exponential splines: Part i-theory and filtering algorithms. IEEE Trans. Signal Process. 53(4), 1425-1438 (2005)

19. H Olkkonen, JT Olkkonen, Measurement and reconstruction of impulse train by parallel exponential filters. IEEE Signal Process. Lett. 15, 241-244 (2008)

20. H Olkkonen, JT Olkkonen, Sampling and reconstruction of transient signals by parallel exponential filters. IEEE Trans. Circuits Syst. Express Briefs 57(6), 426-429 (2010)

21. T Kloos, J Stockler, M Ckler, Zak transforms and gabor frames of totally positive functions and exponential b-splines. J Approximation Thoery 184, 209-237 (2013)

22. M. Rudelson, R. Vershynin. Sparse reconstruction by convex relaxation: Fourier and gaussian measurements. Information Sciences and Systems, 2006 $40^{\text {th }}$ Annual Conference on, IEEE, 2006: 207-212.

23. K. Grochenig. Foundations of time-frequency analysis. Boston, Springer. (2001)

24. HG Feichtinger, On a new segal algebra. Monatshefte fr Mathematik 92(4), 269-289 (1981)

\section{Submit your manuscript to a SpringerOpen ${ }^{\circ}$ journal and benefit from:}

- Convenient online submission

- Rigorous peer review

- Immediate publication on acceptance

- Open access: articles freely available online

- High visibility within the field

- Retaining the copyright to your article

Submit your next manuscript at $>$ springeropen.com 